\title{
CONOCIMIENTOS PROFESIONALES DE LOS PROFESORES
}

\author{
BROMME, R. \\ Institut für Didaktik der Matematik. Universität Bielefeld.
}

Versión española: Gonzalo Guijarro Puebla

\begin{abstract}
SUMMARY
Strangely enough, professional knowledge of teachers has been rarely studied so far. This paper presents starting points for this subject and possible open lines for research are suggested.
\end{abstract}

\section{INTRODUCCION}

Los conocimientos profesionales de los profesores han sido hasta ahora asombrosamente poco estudiados. De cualquier modo entre los profesores está extendida la opinión de que sus conocimientos profesionales no constan únicamente de lo que una vez aprendieron en su formación teórica. Así, pues; se dice a menudo a los enseñantes que comienzan su carrera profesional que su saber teórico no es especialmente útil para la práctica cotidiana. ¿Qué papel juega entonces el saber teórico y de qué tipo son los conocimientos profesionales?

Para una mejor comprensión de las dificultades inherentes a la aplicación de los conocimientos científicos es deseable disponer de conocimientos acerca de en qué consiste el saber profesional de los enseñantes y cómo éste se relaciona con los conocimientos de la asignatura, de la pedagogia y de la psicología. Es también deseable disponer de informaciones sobre la naturaleza psicológica de los conocimientos profesionales. Estos conocimientos sólo pueden hacerse conscientes con esfuerzos.

Determinados conocimientos se tienen más bien «en la punta de los dedos» que en la cabeza. Esto no debe tomarse como una deficiencia, sino como una caracteristica de los conocimientos profesionales. Para otras profesiones, como por ejemplo médicos, arquitectos o ingenieros, también sería esto válido.

En este trabajo se dan puntos de partida para este tema y se seivalan posibles lineas abiertas de investigación.

Tras un ejemplo introductorio se considera en la primera parte la pregunta: ¿Qué se puede esperar de los conocimientos teóricos en la práctica y qué relación mantienen con los conocimientos profesionales?

Para empezar, un ejemplo de lo que en realidad se quiere decir con conocimientos profesionales. Oficia aquí como la noción de los conocimientos que los ensefian- tes usan en su práctica cotidiana. Como en el oficio de los enseñantes se trata de transmitir conocimientos, es por una parte la herramienta del trabajo diario. Pe ro la noción conocimientos profesionales incluye también los conocimientos científicos necesarios para è ejercicio de la profesión, que son los que estructuran el proceder del profesor.

El ejemplo: Un profesor de matemáticas de una escuela primaria observa que algunos alumnos fallan en deter * minadas sumas. Identifica la causa como dificultad en sumar cifras cuyo resultado supera la decena y decide interrumpir el desarrollo del tema por un tiempo para practicar estas sumas. Para ello propone ejercicios es pontáneamente formulados en los que, como por ejemplo en 82 más 33 , solo hay que sumar llevando una vez. Según su experiencia los alumnos comprenden más fácilmente estos ejemplos que cuando hay que sumar lle vando dos veces, como por ejemplo en 86 más 27 . Sabe también que con números pequeños los alumnos en realidad no suman, sino que recuerdan sumas de dos numeros lo que les permite evitar la aplicación de las reglas para sumar llevando. Por ello escoge números que los alumnos ya no pueden dominar mediante el mero recuerdo espontáneo de sumas de dos sumandos. Cuando tras esto algunos alumnos tienen todavia dificultades, termina con la práctica de la regla e intenta aclarar su sentido formando montones de 10 canicas y reuniéndolos.

Estas decisiones suponen un considerable saber profe sional. Por una parte, naturalmente, el conocimiento teórico del algoritmo de la suma y el conocimiento didáctico sobre el tipo de regla que se ha introducido en la clase (anotando las decenas que uno se «lleva»). Por otra parte conocimientos profesionales sobre los errores de los alumnos puesto que el profesor los reconoce como sistemáticos y no los interpreta como errores por descuido o negligencia. Estos conocimientos ya no son 
el objeto de la clase sino un presupuesto de la actividad del profesor. A esto se añade una concepción didáctica de tipo básico: ¿Es útil fijar una convención determinada para la notación de las decenas que uno «se lleva» y presentarla a los alumnos tan inamovible como quizá una regla fundamentada matemáticamente? Esta decisión no se toma en cada caso, sino que es, por supuesto, un presupuesto de la actividad práctica. Además es necesario un conocimiento profesional para juzgar si merece la pena internmpir el curso de la clase. ¿Puede asumirse que los alumnos probablemente pierdan el hilo y al final de la clase ya no sepan si el tema central eran las sumas de números cada vez mayores o las sumas llevando ¿Hay que suponer beneficiosa esta interrupción? ¿Es posible corregir durante esa sola hora de clase ese fallo recién descubierto? ¿Qué consecuencias trae esto para la materia que se estaba impartiendo en principio? ¿Queda suficiente tiempo para ella? Finalmente, ¿qué debe hacerse para no molestar ni aburrir a los alumnos que ya dominan este tipo de suma?

Todas estas decisiones presuponen conocimientos profesionajes. No es necesario tomarlas de nuevo cada vez, sino que pueden ser tomadas de modo rutinario o también venir determinadas por la forma en que se plantea la clase. Incluso el orden en que se sientan los alumnos influye, por ejemplo, en si estas tareas de recuperación pueden o no realizarse paralelamente al tema central de la clase.

El saber profesional incluye por tanto elementos teóricos y consta de reglas empíricas y experiencia práctica. $Y$ aún hay otro aspecto del conocimiento teórico que juega también un papel, a saber: el metaconocimiento sobre el conterido de la asignatura. En el saber profesional se incluye, por tanto, también teoria junto a la experiencia práctica.

No obstante es necesario explicar con claridad la diferencia entre estos conocimientos profesionales y los conocimientos teóricos, tal y como se desarrollan en las disciplinas básicas y se aprende durante la formación como enseñante.

\section{ESTATUS LOGICO-CIENTIFICO DE LOS CONOCIMIENTOS PRACTICOS}

Para aclarar la naturaleza especial de los conocimientos profesionales en contraste con los teóricos resulta útil poner de manifiesto las frecuentes expectativas que han sido establecidas a partir de nuestras propias experiencias en coloquios con enseñantes y científicos. No son el resultado de una encuesta representativa ${ }^{(1)}$, pero pueden encontrarse muy frecuentemente.

\section{Expectativas de los enseñantes respecto a las teorias:}

Las teorias deben contemplar la totalidad de la situación vital en la escuela.
- Por el contrario éstas dividen la situación del profesor en asignaturas, experimentos, planteamientos de cuestiones, etc.

El proceder en la escuela deberia basarse y llevarse a cabo a través de estudios científicos.

- Por el contrario la práctica de la enseñanza no está fundamentada teóricamente sino que implica que cada uno vaya abriendo su propio camino y así es también como es vivida (con mala conciencia) por el profesor.

A partir de estudios científicos deberían poderse de ducir criterios prácticos.

- Por el contrario los investigadores señalan la provisionalidad de sus estudios y se niegan a tales deducciones.

Las teorias científicas deberian estar formuladas con claridad y sencillez.

- Por el contrario una terminología complicada y en muchas materias un excesivo formalismo obstaculizan la comprensión.

Estas frustaciones en el aprovechamiento práctico de las teorías tienen seguramente en parte su causa en un inadecuado o incomprensible planteamiento de las investigaciones y sus resultados.

En este trabajo no se pretende dar respuesta a las mencionadas expectativas frustradas respecto al aprovechamiento práctico de los conocimientos teóricos. La posible validez de esta respuesta es seguramente distinta para cada materia. En nuestro contexto nos ocuparemos de otro aspecto de las mencionadas expectativas. Las expectativas son expresión de una cierta epistemología sobre la relación entre conocimiento cientifico y conocimiento práctico. Esta epistemologia del conocimiento práctico está ampliamente extendida y alienta ese tipo de expectativas. Pero es, desde un punto de vista científico-teórico, muy problemática.

Las expectativas de una aplicación de las teorías de las correspondientes ciencias empíricas, que directa o indirectamente investigan temas escolares relevantes, han sido alimentadas por puntos de vista científico-teóricos, según los cuales la práctica diaria con fundamento científico es, en principio, como si se experimentara. El trabajo del enseñante al aplicar conocimientos teóricos se entendería como análogo al del científico que saca conclusiones de las teorias y las comprueba.

Este modelo -el sociólogo Schön (1983) lo describió como el modelo del racionalismo técnico ${ }^{(2)}-$ se desa" rrolló principalmente en el racionalismo critico (por ejemplo, Prim \& Tíman, 1973).

En la psicología (Bromme \& Hömberg, 1986; Herr" mann 1979; Krapp \& Heiland, 1986) y en las ciencias de la educación (Drerup \& Terhart, 1979), sin embar* go, se desarrolla otro punto de vista en relación con la recepción de los trabajos del teórico de la ciencia Bunge (1967, II). A raiz de estos trabajos quedan los 
fundamentos de la práctica cotidiana en una relación muy vaga con las teorias científicas.

No vamos a repetir aqui esta argumentación científicoteórica. Fundamentalmente afirma que las teorias científicas son enunciados generales sobre hechos empiri. cos y sus contextos. Estos enunciados o sistemas de afirmaciones son necesariamente generales frente a las concretas circunstancias del contexto bajo las que aparecen los fenómenos que son descritos y explicados a tra* vés de los enunciados. Gradualmente ignoran estos enunciados las circunstancias concretas del contexto.

Unos conocimientos aplicables deben por el contrario referirse a situaciones concretas, deben contener elementos normativos sobre objetivos que hay que alcanzar y deben precisamente evitar las generalizaciones y llenar de contenido concreto los conceptos abstractos que deban aplicarse. (Aquí debe advertirse sobre un malentendido: También la producción y no solo la aplicación de conocimiento científico viene determinada por el establecimiento de objetivos que hay que alcan. zar. Pero éstos no son parte de las propias teorias científicas. En el conocimiento relacionado con su aplicación práctica, los objetivos son, por el contrario, parte de este conocimiento, por ejemplo las expectativas sobre resultados deseados en la práctica cotidiana).

La concreción de las circunstancias del contexto com. prende también la contemplación de los objetivos y las reflexiones de las personas implicadas. Algunos enunciados científicos de los sociólogos dejan de lado precisamente estas reflexiones de las personas sobre las que trabajan. Este tipo de abstracción debe revocarse para la aplicación de los enunciados científicos,

Por ejemplo, cuando se utilizan hallazgos científicos para la motivación de los alumnos, hay que tener en cuenta si estos reflexionan sobre ellos y comprenden que deben ser motivados. El éxito de una aplicación de este tipo deberfa estar notablemente influida por es. to. La transformación de un enunciado científico en una pieza de conocimiento aplicable -Bunge (1976, II) habla aquí de reglas tecnológicas- no es por tanto, lógicamente enfocado, una deducción. No se com. prueba la validez actual de un enunciado general del tipo «Si... entonces...» para un caso concreto, sino más bien se interpreta de nuevo una relación general de este tipo a la luz del caso concreto en que haya de aplicarse y en relación con el establecimiento de objetivos que hay que alcanzar.

Esta concreción es, por ello, no una pura y simple conclusion, pues los enunciados científicos están normalmente especializados por disciplinas. La labor de los profesionales tiene lugar, sin embargo, en contextos concretos que vienen dados en su globalidad. Por tanto hay que revocar gradualmente esta subdivisión de la realidad. Los conocimientos de los profesionales son, por tanto, a menudo eclécticos (Schwab, 1971) y con. tienen elementos procedentes de diferentes disciplinas, cuando los problemas que hay que resolver atraviesan varias disciplinas.

Los conocimientos prácticos son pues, desde un enfoque lógico, de otro origen que los resultantes de la la. bor de científicos básicos. Los conocimientos teóricos requieren una transformacion heurística y una integración para poder devenir conocimientos prácticos. Las circunstancias concretas de semejante transformación son naturalmente distintas, según las disciplinas científicas y sus campos de aplicación.

Esta transformación tampoco tiene que ser emprendida por un profesional aislado. Más bién hay por ejemplo en las materias que son de interés para el trabajo del profesor, como pedagogía, didáctica y también psicologia, puntos de partida que quieren producir de inmediato saber tecnológico por sí mismas. De cualquier modo, dudamos si este requerimiento es un principio realizable. Siempre resta una parte del trabajo de transformación e integración para el profesor. Por tanto hay que mantener una diferencia básica, lógicamente fun* damentada entre teorias científicas y conocimientos prácticos. Así pues, las antes mencionadas expectativas respecto a las teorías científicas no pueden ser en principio satisfechas por ellas. Estas expectativas resultan al mismo tiempo instructivas, porque reflejan en realidad las características psicológicas de los conocimientos prácticos.

La diferencia lógicamente fundamentada entre conocimientos teóricos y prácticos no libera a los científicos de la necesidad de desarrollar relaciones de intercambio referentes a la práctica (escolar). Es de suma utilidad diferenciar entre el contenido y el lado social de la relación entre ciencia y práctica. Para la formación y perfeccionamiento de los profesores, pero también para la investigación, es importante una intensa relación entre investigadores y profesores, pero estos aspectos sociales de la relación teoría-práctica no son aquí nuestro tema. De cualquier modo las concepciones sobre los conocimientos profesionales para la estructuración de la mencionađa relación, son importantes. La aceptación, lógicamente fundamentada, de la independencia de los conocimientos cientificos por un lado y de los conocimientos profesionales de los profesores por otro, sólo puede ser de utilidad a la rela. ción (social) entre profesores e investigadores, porque protege tanto a unos como a otros de expectativas imposibles de satisfacer. El conocimiento de la naturale. za de estos conocimientos profesionales puede fundamentar una aceptación recíproca de las diferencias en las tareas de investigadores y profesores.

Una colaboración a la investigación del problema teoría-praxis consiste en examinar los conocimientos profesionales. De esto trata el presente trabajo. El examen de la naturaleza psicologica de los conocimientos profesionales, su estructura y sus contenidos, asi como también de su desarrollo, es un desafío para la investigación empírico-didáctica y psicológica. Como los 
conocimientos profesionales son de naturaleza muy peculiar y debido a la gran variedad de problemas que se presentan en la práctica, ha de ser desarrollado de nuevo cada día. Hay que contar con una «sabiduría de la praxis». Al menos hay que contar con la posibilidad de que durante la investigación de los conocimientos profesionales aparezcan resultados que puedan extraerse y convertirse en aprovechables. La reconstrucción y análisis de tales conocimientos en enseñantes expertos y posiblemente eficaces puede proporcionar datos sobre cómo deben prepararse las teorias para que puedan ser utilizadas como parte de los conocimientos profesionales relacionados con la práctica. Tal investigación es sólo realizable como investigación básica y toca preguntas básicas de la psicología y pedagogía, por ejemplo referentes a la relación entre conocimientos y prácticas, la génesis de las competencias o las relaciones entre asignatura y método didáctico.

\section{LA INVESTIGACION COGNITIVA SOBRE LOS PROFESORES}

En los últimos 10 años se ha desarrollado la investigación cognitiva sobre profesores. En este campo investigativo se examinan los procesos y estructuras del tratamiento de información por los profesores y en relación con su trabajo de clase y preparación de la misma.

Los puntos de partida metodológicos y las orientaciones teóricas en este campo son muy diversas. Pero en la base de estos trabajos subyace la idea de que la actuación de los profesores depende notablemente de cómo interpretan su entorno escolar, qué metas persiguen y como aprovechan y califican las informaciones que se ponen a su disposición. (Ver Ben-Peretz, Bromme \& Halkes, 1986, Calderhead, 1984, Clark \& Peterson, 1986, Hofer, 1986, Villar Angulo, 1986).

Un ejemplo de planteamiento de preguntas y de la eficacia de este campo investigativo es el estudio de la preparación de clases en profesores experimentados. El punto de partida fue la observación de que los profesores con experiencia no aplican ninguna de las teorias sobre preparación de clases que se enseñan como modelos, sino más bien desarrolian sus propias estrategias para el cumplimiento de esta tarea. Estudios empíricos mostraron que la preparación de clase se lleva a cabo mediante una concentración en las actividades de los alumnos con la asignatura (elección de ejercicios) y a través de una planificación de tiempo y contenidos. (Yinger, 1980, Bromme 1981, Marcelo, 1986). Con eso no se actúa según exigen los modelos teóricos u orientados al aprendizaje por objetivos. La planificación no comienza por tanto, p.e., con el análisis de objetivos. Naturalmente podemos no tomar necesariamente como prescripción para la formación de profesores el tipo de planificación de los profesores experimentados; pero ésta constituye la base para alcanzar recomendaciones realistas y modelos sencillos de pre- paración de clases para principiantes (por ejemplo Meyer, 1980, Bromme \& Seeger, 1979).

Para nuestra pregunta respecto a los conocimientos profesionales resulta de interés un cierto punto de partida dentro de la investigación cognitiva sobre profesores: El profesor como experto. Partiendo de esta idea, buscariamos profesores experimentados y a ser posible eficaces y describiníamos empíricamente su pensamiento y conocimientos.

En parte los comparariamos con el pensamiento de profesores sin experiencia laboral (Principiantes con poca experiencia profesional) (Berliner, 1986, Bromme \& Brophy, 1986).

Este modelo de ver las cosas fue sugerido por los estudios sobre modos de pensar y conocimientos de expertos en otros campos profesionales de parecida complejidad y dificultad. Así, hay estudios sobre las estrategias de los métodos al diagnosticar enfermedades, las decisiones de los agentes de bolsa o el pensamiento de los ingenieros. Igualmente sugerentes fueron los estudios en que se comparaba el modo de enfrentarse a complejos problemas de física de estudiantes y profesores (Chi \& Glaser, 1986).

A pesar de las diferencias existentes entre estos campos profesionales, se encontraron parecidos en el «saben de los correspondientes expertos frente a los principiantes y los profesionales de menor experiencia.

Los expertos disponen de mayores conocimientos específicos sobre el campo profesional que los principiantes. Los conocimientos de los expertos se refieren a relaciones «subterráneas», esto es a relaciones entre sucesos y sus causas y consecuencias. Los expertos consideran lo esencial de un problema y no recuerdan demasiado los detalles, sino las relaciones funcionales importantes del planteamiento de un problema. Este enfoque resulta económico bajo el punto de vista de las correspondientes propuestas de tareas u objetivos.

A primera vista, estas características de los conocimientos de los expertos parecen poco reveladores ¿No perfilan simplemente las capacidades y conocimientos de personas inteligentes? No resulta dificil darse cuenta de que no es así, si pensamos que de níngún modo disponen los expertos en su conjunto de unos mayores conocimientos, ni son más inteligentes en general, ni resuelven siempre los problemas más deprisa que resolutores de problemas sin experiencia. Abreviando, se puede resumir asi la diferencia entre expertos y principiantes: Los expertos disponen no de más conocimientos sino de otros conocimientos que sus colegas menos experimentados. Esos conocimientos distintos están estructurados de modo adecuado a la exigencias del entorno y se muestran en parte a traves del dominio de procedimientos y también en rutinas y no en los conocimientos reproducibles (proposicionales).

A partir del concepto de experto, se examina, pues, particularmente el enfoque dado a los problemas y los co- 
nocimientos utilizados en tareas complejas. Así mismo característico de este punto de partida es el reconstruir en relación con su trabajo concreto el pensamiento y conocimientos del experto (Bromme, 1987 a).

Un problema hasta ahora no satisfactoriamente resuelto es el de a quién denominamos experto, para posteriormente examinar sus conocimientos profesionales. En muchos estudios se averigua durante un largo período de tiempo los rendimientos de los alumnos de distintos grupos (con exclusión estadística de los conocimientos previos de los alumnos) con lo que se averiguan las diferencias de éxito en el aprendizaje de los diversos grupos. Estas diferencias serán adscritas a las diferencias de calidad en los procederes de los profesores, caso de ser las demás circunstancias comunes. En las investigaciones entre expertos, se trata de determinar qué pensamiento y conocimientos están en la base de esas diferencias. Este proceder es sumamente prolijo y limita el criterio al desarrollo de los alumnos en la materia. En esta via investigativa quedan todavía por estudiar aspectos problemáticos de la idea de experto, por ejemplo la excesiva especialización.

\section{UN EJEMPLO PARA UN ESTUDIO SOBRE «LOS PROFESORES COMO EXPERTOS»}

A continuación se pretende presentar algunos resultados de un estudio sobre la percepción que los profesores tienen de la comprensión de sus alumnos. Esta investigación ha sido ya prolijamente descrita (Bromme, $1987 \mathrm{~b}$ ) y sólo se la menciona ahora para ilustrar el enfoque del estudio sobre expertos. Hemos preguntado de qué problemas de comprensión y de qué progresos de sus alumnos individuales se acuerdan los profesores de matemáticas.

Hasta el momento ha habido pocos trabajos empiricos sobre cómo perciben los profesores la comprensión de sus alumnos. Y éstos muestran más bien una imagen negativa. Según los mismos, los profesores reconocen poco la comprensión de sus alumnos (Jecker y otros, 1965). Shroyer (1978) preguntó a profesores, tras ver conjuntamente cintas de vídeo sobre la clase recién dada, si recordaban algún momento en el que los alumnos hubieran tenido especiales dificultades o hubieran mostrado progresos inesperados. Continuó preguntando lo que los profesores habian reflexionado al respecto y observó paralelamente las clases, encontrando que solo un $3 \%$ de esas dificultades y progresos eran percibidos como tales.

Nosotros hemos escogido un proceder parecido. De cualquier modo nuestras entrevistas se basaban en la descripción de los problemas de matemáticas de la clase y no en la visión de una cinta de vídeo, queriendo evitar con esto observaciones posteriores.

Las entrevistas con 19 profesores de matemáticas de $5^{\circ}$ a $7^{\circ}$ de básica, referida cada una a una hora de cla- se, fueron valoradas en cuanto a contenidos. Se anali$z \sigma ́$ si los profesores recordaban los progresos de aprendizaje o los problemas de comprensión y quién era el protagonista en cada caso: La totalidad de la clase, escolares individualmente nombrados o grupos dentro de la clase. Esta pregunta investigaba la perspectiva del profesor respecto a la comprensión en la clase. Se analizo, pues, con qué frecuencia recuerda el profesor alumnos individuales. El resultado es a primera vista más o menos tan negativo como el del mencionado estudio de Shroyer (1978) (a pesar de las diferencias de método y cuestionario).

La cifra de problemas de alumnos y progresos de aprendizaje recordados es sorprendentemente baja. Por hora, se recordaban en promedio sólo 1.8 casos de dificultades de alumnos individuales. Aparte de esto se encontraron en promedio otras tantas menciones con la totalidad de la clase o un grupo especiffico como protagonista.

Los análisis de las clases muestran, sin embargo, muchos más alumnos con problemas de comprensión, pero de esto se menciona muy poco en los informes de los profesores. Es decir, apenas se hicieron observaciones en este sentido sobre alumnos individuales que se recordaran después.

Con esto no se afirma que la percepción de los profesores sea absolutamente escasa. Los profesores pregun. tados observaron a la clase en conjunto. Para el pro. tagonista "clase», hay observaciones en todos los profesores, mientras que para los problemas de compren. sión de alumnos individuales, 8 de 19 profesores no pu * dieron mencionar ninguno.

Son el conjunto de la clase y el curso de la hora lectiva los que ocupan el punto central de los recuerdos. Y con esto cambia también la imagen de la aparente ceguera frente a la comprensión de la clase. En todos los ca. sos, sólo hubo 162 episodios en el transcurso de la clase que contuvieran problemas de comprensión o progresos de aprendizaje; pero fueron los episodios que correspondieron con el comienzo de nuevos temas del programa. En este sentido eran los episodios clave desde el punto de vista del profesor. Resumiendo puede mantenerse que los casos individualizados se recuerdan cuando son de valor estratégico para el curso del diálogo sobre los contenidos. Con "valor estratégico" que. remos decir que llegan en el momento en que, según el profesor, se «engancha» (esa imagen utilizo una pro* fesora), o donde debe tener lugar el tránsito entre unos conocimientos y otros nuevos. Interpretamos esto co. mo indicio de que es el aspecto general del desarrollo de la hora lectiva en conjunto y no el diagnóstico sobre alumnos particulares lo que ocupa el punto central de la percepción de los problemas por parte del profesor. Esto es importante, porque, en la investigación. de la percepción de los alumnos por los profesores, alumnos y profesores se aislan recíprocamente. Pero al menos en relación con la labor docente parece que 
la percepción de los alumnos tiene lugar desde puntos de vista completamente distintos. No sólo se percibe lo que aprende un alumno individual sino también cuál es su colaboración en la presentación de la materia y para esto es también necesaria la tematización de las dificultades de comprensión de la materia.

Llegamos a esta interpretación partiendo de nuestra reconstrucción de las exigencias sobre el profesor. Si ob. servamos en detalle la tarea que supone dar clase a 25-30 alumnos, resulta claro que los profesores no pueden diagnosticar los fallos de sus alumnos como los médicos diagnostican enfermedades.

Nuestro estudio muestra que aparentes defectos de los profesores - aquí la falta de percepción individual de los alumnos- se muestran bajo una luz muy distinta al efectuar la correspondiente reconstrucción de las exigencias sobre los enseñantes.

Al mismo tiempo, se hace claro que el pensamiento y los conocimientos profesionales de los enseñartes pue den ser tomados en relación con una reconstrucción de las exigencias.

El significado de estos hallazgos y la utilidad del pun. to de partida "expertos» para estudiar las capacidades de los profesores resultan claras cuando uno se representa la complejidad y falta de estructura de las exigencias que pesan sobre ellos.

\section{LAS COMPLEJAS EXIGENCIAS DEL «DAR CLASE»}

Desde el punto de vista psicológico, el dar clase exige (bajo las aquí habituales circunstancias de organización como: asignaturas separadas, duración de la clase prefijada, etc.) la realización, a ser posible paralelamente en el tiempo, de diferentes tareas parciales. Hay que presentar unos contenidos y para ello deben aprovecharse las colaboraciones de los alumnos. La duración de la clase ha de ser respetada. Al mismo tiempo hay que realizar observaciones de los alumnos necesarias para la evaluación y otras para ayudar a los alumnos individuales y además, al mismo tiempo, es necesario mantener debidamente el interés y la colaboración de los alumnos.

Estas exigencias son en parte contradictorias. Así, es recomendable esperar un cierto tiempo tras efectuar preguntas a la clase hasta que algunos se ofrezcan a contestar, si empiezan a decir algo tartamudeando con. tinuar esperando hasta que consigan formular su respuesta. Al mismo tiempo es imprescindible una cierta fluidez en el transcurso de la clase para mantener la atención de los demás alumnos (Shulman, 1986).

La exposición de los contenidos de la materia exige a menudo decisiones ad hoc sobre la bondad de los ejemplos e ilustraciones alternativas, cuando resulta evidente que los alumnos no han entendido algo. Más aún, es necesario reconocer y aprovechar las posibles relaciones entre distintas partes de la asignatura. Así, por ejemplo, en la clase de matemáticas muchos temas ofrecen de nuevo elementos de las operaciones con fracciones. Muchos alumnos tienen tendencia a parcelar sus conocimientos y a no reconocer la necesidad de aplicar algunos de ellos cuando «no tocan». Para practicar esto, resulta útil reconocer estas relaciones y lla. mar la atención de los alumnos sobre ello. De cualquier modo, tampoco conviene exagerar, o de lo contrario los alumnos perderán el concepto de lo fundamental y nuevo en la lección que se esté explicando. El dar clase exige así mismo juzgar cuál es el ritmo adecuado, es decir, el equilibrio entre las velocidades de aprendizaje de diferentes alumnos. Es sabido desde los estudios de Kounins (1970) referentes al modo de dirigir la clase, que los problemas de disciplina se minimizan cuando son captados en ciernes y solucionados sobre la mar. cha. Esta estrategia no es siempre adecuada y depende del reconocimiento del momento justo en que es necesaria la toma de una medida abierta y que cuesta tiempo.

Estos pocos ejemplos deben bastar para aclarar que las exigencias profesionales que suf ren los enset̃antes son de parecida complejidad y dificultad que las de, por ejemplo, un ingeniero o un médico. Esta compleja tarea de dar clase exige unos conocimientos específicos, en cuanto que la experiencia y la teoría se encuentran integradas y a través de las exigencias son percibidas e interpretadas subjetivamente (Schönwälder, 1983, Veenmann, 1984).

\section{CONOCIMIENTOS PROFESIONALES: ASPECTOS DE CONTENIDO}

A primera vista, los conocimientos profesionales parecen quedar suficientemente descritos a través de «conocimientos de la asignatura», y "pedagogía», así como "didáctica de la asignatura». Efectivamente así es, pero debemos continuar desentraniando estos campos del saber si queremos comprender la relación entre conocimientos profesionales, manejo de la clase y presión del oficio. El contenido de la asignatura tiene un especial significado, ya que no sólo es un presupuesto básico, sino al mismo tiempo también un objeto de los manejos del profesor. A continuación se escogen las matemáticas como ejemplo.

Las matemáticas constituyen un buen ejemplo para nuestra argumentación porque parece ser la asignatu* ra que menos interpretaciones del profesor requiere. En esta asignatura, los conocimientos profesionales sobre contenidos y didáctica de la materia parecen ser especialmente claros y explícitos, fundamentados en la iógica de las reglas y conceptos matemáticos. Pero, como muestra una observación más minuciosa, no es éste el caso necesariamente. Por esto, en el ejemplo de las matemáticas es en el que más dificil resulta demos- 
trar, pero también en el que esta demostración es más categórica, que tras el «contenido de la asignatura» se esconden unos conocimientos profesionales independientes.

Siguiendo en parte una propuesta de Shulman (1986), diferenciamos entre:

- Conocimientos de matemáticas. $\mathrm{E} 1$ profesor los aprende en la carrera y abarcan entre otras cosas principios matemáticos, reglas y modos de pensar y técnicas matemáticas.

- Conocimientos curriculares. Están descritos en Ios planes de estudio y codificados en los libros de texto y otras herramientas didácticas. Comprende también el conocimiento de las herramientas alternativas existentes, así como conocimientos de contenidos de otras asignaturas que puedan traerse eventualmente a colación en la clase de matemáticas.

- Conocimientos sobre la clase. La materia explícitamente transmitida por el profesor en la clase representa ciertamente una selección de los programas, si bien la programación general del centro determina el marco de tal selección. Dependiendo del desarrollo del curso académico, va modificándose esta programación y de ello resultan los conocimientos efectivamente impartidos. Este corpus de conocimientos no es sin embargo una mera parte del programa oficial, ya que no se forma simplemente por selección. EI conocimiento de la clase aparece mediante el establecimiento de relaciones y un especial equilibrio a la medida de las especificas circunstancias de la clase. Por ejemplo, las dadas por los requisitos de acceso a niveles superiores. El conocimiento de la clase incluye también materia que sólo se enseffa de modo implícito. Así por ejemplo, se trabaja extensamente en matemáticas con los signos de igualdad antes de tratar explícitamente de su significado. Los profesores se dan cuenta con frecuencia de la existencia de estos conocimientos, al notar que los alumnos no disponen de ellos. Se da por supuesto, pero no se habla de ello (Chevallard, 1985).

- Conocimientos sobre lo que los alumnos aprenden. Para dar clasé con efectividad, el profesor debe tener también conocimiento de lo que los alumnos han entendido y retenido. Lo realmente aprendido por los alumnos no es una simple parte de lo expuesto en la clase sino que más bien, como muestran estudios empíricos sobre la comprensión de las matemáticas por los alumnos, éstos construyen al menos en parte sus propios algoritmos que les permiten salir del paso en clase, pero que les resultan un obstáculo al pretender progresar en la materia (Brousseau, 1986.., Gil \& Carrascosa, 1985).

Los errores de los alumnos y sus dificultades de comprensión no son en estos casos simples lagunas. Para el profesor, es por tanto necesario conocer en lo posible esta comprensión de los alumnos, para poder proceder consecuentemente en clase.
- Metaconocimientos. Pero iqué estructura el trato con este tipo de conocimiento? ¿Qué posibilita una cierta coherencia, qué permite el mantenimiento de tan diferentes tipos de informaciones como las aquí mencionadas? Es conocimiento sobre la naturaleza de Ios conocimientos respecto a la escuela y la asignatura, respecto a los fines y objetivos que han de conseguirse.

Los metaconocimientos definen por tanto el marco de orientación en el que se valoran los conocimientos y su relación con la propia profesión. También podemos definir el metaconocimiento como la filosofía del profesor en cuanto a las matemáticas y la enseñanza. Pero el metaconocimiento tiene, a pesar de su carácter filosófico, muy concretos efectos sobre la práctica didáctica.

Un ejemplo: Los conocimientos matemáticos pueden entenderse esencialmente como sintaxis para la formalización de afirmaciones en las que no influyen sus relaciones fuera de las matemáticas. Pero también se puede ver la importancia de los conceptos matemáticos en su relación con la realidad abiertamente matemática. Ambos enfoques reflejan diferentes posiciones en la controversia básica de la matemática. No pueden calificarse de correctas o erróneas. Tales enfoques influyen también en las consideraciones que se hacen cara a la transformación del programa oficial en el conjunto de la materia realmente impartido. Deberían asimismo decidir qué fallos y malentendidos de los alumnos deben juzgarse de importancia y necesaria corrección y cuáles, en interés del avance en los programas, pueden tolerarse.

En un estudio de casos Thomson (1984) analizó los enfoques matemáticos de tres profesores y sus efectos en las clases. La profesora $J$. consideraba las matemáticas como un sistema lógico, que existe independientemente de los propios conocimientos. A partir de ahí extraía también sus principios didácticos. Consecuentemente, entendía su labor como la presentación clara y consistente de los contenidos de la asignatura y esperaba ante todo de sus alumnos que aprendieran las relaciones entre lo ya explicado y la nueva materia. La profesora $\mathrm{K}$. tenía por el contrario un concepto de la asignatura orientado a los procesos y por tanto, la estructura de sus clases se dirigia al desarrollo de descubrimientos por parte de los alumnos.

Los enfoques en cuanto a contenido, su importancia y posibilidad de realización no tienen, sin embargo, que coincidir necesariamente con la práctica cotidiana en clase. Los metaconocimientos matemáticos parecen exigir de algunos profesores una práctica cotidiana muy determinada, pero ellos mismos comprenden que ésta no es realizable. Esto es especialmente cierto cuando un profesor sospecha que la claridad y comprensibilidad de sus clases solo son alcanzables mediante la acentuación de los aspectos formales de la matemática. Lo que desde el punto de vista de la ciencia matemática aporta claridad no es suficiente para facilitar la comprensión de los alumnos. 
Sospechamos que en semejantes discrepancias estriba una notable fuente de tensiones profesionales. Ese sería por ejemplo el caso cuando los profesores toman los criterios de la disciplina cientffica que imparten $\mathrm{co}$ mo única orientación para sus clases. Cuando entonces la clase resulta incomprensible para los alumnos, se producen tensiones emocionales. Por tanto es importante reconocer las diferencias relativas a los diversos tipos de conocimientos.

También hay modelos didácticos, como por ejemplo el llamado de desarrollo mediante preguntas, que coinciden a menudo con ciertos enfoques matemáticos sobre la materia a impartir.

- Conocimientos sobre didáctica de la asignatura. La estructura lógico-matemática de la asignatura no permite por sí misma decisiones. Para encontrar formas válidas de presentar la asignatura, para determinar la sucesión temporal de los temas, para sopesar qué temas han de tratarse más intensivamente, son necesarios conocimientos de didáctica de la asignatura. Estos conocimientos tienen un carácter especial. Son mixtos en cuanto que informaciones psicológicopedagógicas y experiencias del propio profesor se aúnan con los conocimientos matemáticos. Esta mezcla supone la transformación de la estructura lógica de los contenidos en una sucesión temporal. Supone además la necesidad de cambiar la estructuración e importancia de conceptos y reglas. Lo que desde el punto de partida de la teoría matemática ocupa un puesto central, tiene desde la perspectiva didáctica menor importancia.

$\mathrm{La}$ antes mencionada transformación de los conocimientos teóricos supone la mezcla de informaciones de distintos tipos. Así, por ejemplo, exige la evaluación de la dificultad de una tarea, una integración de informaciones relativas a la asignatura y a los alumnos. También el aclarar a los alumnos sus dudas exige esas integraciones de informacion de diversos origenes (Bromme \& Juhl, 1987). Naturalmente, las explicaciones relativas a los contenidos y las relativas a los alumnos no son mutuamente excluyentes. Circunstancias psicológicas, como motivación o colaboración, se realizan a través del trato de los alumnos con los contenidos. Pero proporcionan diferentes puntos de partida acerca de cómo reaccionar ante problemas de comprensión de ejercicios. Es de suponer que aquí también sean de importancia los metaconocimientos, es decir la filosofía del profesor respecto a las matemáticas y su enseñanza. Que sepamos, no existen hasta ahora herramientas conceptuales para caracterizar ese aspecto mixto de los conocimientos sobre didáctica de la asignatura. Por eso no es sorprendente que los profesores no lo consideren una cualidad importante de sus conocimientos de didáctica de la asignatura y lo supediten a otros tipos de conocimientos como los teóricos o pedagogicos a la hora de tomar decisiones.

- Conocimientos pedagógicos. Nos referimos al campo de los conocimientos que son validos con relativa independencia de la asignatura. Comprendería determinados aspectos metodológicos de la clase, el proceder con niños de educación difícil y asimismo la organización del centro escolar. En este contexto, hay que señalar que también aquí se trata de normas y metas que se manifiestan más bien como actitudes que como conocimientos verbalizables sobre hechos o reglas. No nos ocuparemos aquí en profundidad de este campo de conocimiento, no porque no sea importante para la práctica profesional sino porque es sabido que este campo de conocimiento supone una condición previa para la capacidad profesional y no simplemente un objeto que ha de transmitir el profesor; lo que estaba por demostrar en cuanto a los conocimientos de la asignatura.

\section{CONOCIMIENTOS PROFESIONALES: AS- PECTOS PSICOLOGICOS}

Con aspectos psicológicos, nos referimos a las caracteristicas de las disponibilidades mentales (consciencia), de la "verbalizabilidad» y a las posibles formas de representaciones mentales de conocimientos. Los conocimientos científicos se presentan, según sabemos, en forma de afírmaciones teóricas y reglas metodológicas. Iunto a esto, se necesitan las suposiciones tácticas que en la teoria cientifica se describen también como suposiciones paradigmáticas. El conocimiento científico está - según pretende - libre de contradicciones en el seno de una teoria y fundamentado de modo explícito y es aplicable a la práctica. El conocimiento cotidiano presenta por el contrario otras caracteristicas: A menudo sus "usarios" no son conscientes del mismo, solo es parcialmente verbalizable y no está libre de contradiccion.

¿Qué caracteristicas presentan los conocimientos profesionales que puedan describirse como una mezcla de conocimientos científicos y conocimientos adquiridos mediante la experiencia práctica (conocimientos escolares cotidianos)?

La siguiente presentación de algunas características no es ni exhaustiva ni completa; pero quizás basta para aclarar por qué los profesores, con frecuencia, no son conscientes de la extensión y en algunos casos tampoco de la calidad de sus conocimientos profesionales.

Los conocimientos profesionales (como también los conocimientos cotidianos y las suposiciones tácitas paradigmáticas del conocimiento científico) no son fácil. mente verbalizables por sus poseedores. Se puede discutir si tiene sentido siquiera hablar de conocimientos. Hay muchos investigadores que en rechazo al filosofo Polanyi (1964) usan en este contexto el concepto de «saber mudon (tacit knowledge). Además, no se trata aquí de voluntarias falsas informaciones, como por ejemplo en una encuesta. Esa posibilidad está completamente excluida. 
Una dificultad estriba, más bien, en falsas informaciones involuntarias, debidas al deseo de proceder de modo racionalmente fundamentado.

Otra dificultad se encuentra en la construcción de procederes conscientes sobre un sistema de rutinas. El dar clase exige la realización paralela de una multiplicidad de tareas parciales (ver arriba, sección 3 ). Para lograrlo, una cierta parte ha de llevarse a cabo mediante rutinas y así mantener libre la atención consciente para los problemas no solucionables de modo rutinario. Debido a esto, los profesores novatos piensan durante la clase en cuestiones de organización de la clase y motivación de los alumnos, mientras por el contrario, los profesores experimentados piensan en los contenidos de la asignatura y las colaboraciones de los alumnos. La organización del transcurso de la clase y el mantenimiento de la atención de los alumnos se realizan de modo nutinario. No obstante, también esto requiere conocimientos profesionales, pero se encuentran difuminados en el ágil proceder del profesor (Bromme \& Brophy, 1986). Si se quiere uno representar ese saber profesional es necesario tomar distancia. Por ejemplo a través de una cinta de vídeo.

Los conocimientos profesionales pueden estar en la base de una actuación sin que uno se haya percatado nun ca de poseerlos. También es posible adquirir algunos modos de proceder mediante imitación, pero sin ser consciente de su importancia y lógica.

Esto es válido, por ejemplo, para partes de la programación no explícita. El proceder profesional, sigue en parte una lógica no necesariamente consciente para sus usuarios. Un ejemplo es el rígido reparto de los contenidos en asignaturas. El principio de asignaturas permite la práctica del pensamiento especializado de cada disciplina, pero dificulta la visión de conjunto que sólo puede lograrse por medio de esfuerzos conscientes o de muy determinados pasos organizativos, como la clase por proyectos (Centros de interés).

El profesor no ha de ser necesariamente consciente de este principio durante la preparación de las clases, aunque es efectivo que lo sea. Es sin embargo posible liegar a ser consciente de este principio para el trabajo profesional a través de los correspondientes instrumentos conceptuales.

También la experiencia forma los modos de actuar, sin que por ello haya que formularla conscientemente como regla. El ejemplo de las capacidades manuales podemos aclararlo. La descripción del arte de un alfarero no se agota en una regla o conocimiento sobre la elaboración de cacharros de barro.

Debido a estas características psicológicas de los conocimientos profesionales, consideran algunos el «dar clase" como un arte. Este arte no sería accesible a un análisis científico o por lo menos no sería alcanzable por mera formación científica, (respecto a este debate, Gage, 1979). Pero la práctica del profesor se lleva a cabo de un modo mucho más verbal que la del alfarero. Los medios de trabajo del profesor son asimismo de carácter más conceptual (aun cuando haya también una gesticulación no verbal).

Por esto, los conocimientos que subyacen a estos procederes pueden hacerse conscientes, al menos en principio.

- Los conocimientos profesionales tienen propiedades que en psicología se describen como características de "esquemas» y "scripts» (3).

Hay que resaltar dos caracteristicas de los conocimientos humanos: El conocimiento sobre un hecho o una situación abarca las características notables y deja las demás.

Gracias a esto es aplicable a nuevas situaciones (los psicólogos mencionan a menudo en este contexto el eiemplo del esquema de un restaurante. El cliente dispone de conocimientos generales sobre el curso de los acontecimientos en un restaurante, lo que le posibilita comportarse adecuadamente donde no había estado nunca antes). La segunda característica se refiere a la variedad de conocimientos sobre la situación específica. El esquema del restaurante, por ejemplo, contiene un esquema de elección de comidas, y éste a su vez un esquema de estudio de la carta. Podemos procesar ahora grandes cantidades de información, porque basta con usar esas informaciones cuando llega el momento. Basta, por ejemplo, para planificar un día, proponerse ir a comer a un restaurante. No es necesario activar el esquema "encargar la comida» por estar incluido en el anterior.

Estas descripciones de los conocimientos humanos han mostrado, desde luego, sólo la realidad psicológica de algunas características de los conocimientos humanos, que ya eran ampliamente conocidas desde el punto de vista filosófico. Así, por ejemplo, el concepto de variable contiene la idea de substituibilidad en situaciones especificas del conocimiento. Pero las pruebas empíricas de la estabilidad y al mismo tiempo de la flexibilidad del conocimiento humano parecen todavía muy prometedoras para el análisis de los conocimientos profesionales.

Por ejemplo, la percepción e interpretación del transcurso de la clase se rige por estos esquemas. Los profesores experimentados tienen diferentes esquemas que los principiantes y ellos les posibilitan juzgar más rápido si las respuestas de los alumnos son adecuadas para la continuación de la clase. Estos esquemas permiten asimismo diferenciar distintos tipos de ruido en el aula (Calderhead, 1984).

Wahl (1983) diferencia entre enfoques de la situación y de los procedimientos en los profesores. Consiguió mostrar empíricamente, con ayuda de situaciones con problemas de disciplina o progresos de aprendizaje en alumnos, que los profesores desarrollan modelos individuales de interpretación de las situaciones. Esta in- 
terpretación de la situación seguia a determinados tipos de situaciones, en cada uno de los cuales existian unas pocas alternativas de acción. La estrecha relación entre enfoque de la situación y decisión entre pocas alternativas de acción se hace evidente cuando se actúa bajo gran presión. Pueden interpretarse estos hallazgos en el sentido de que los esquemas de la situación y las posibilidades de acción están estrechamente relacionadas en los conocimientos profesionales del profesor (Hofer, 1986).

En la investigación cognitiva sobre profesores, se aprovecharon en principio, como punto de partida, estos conceptos para la caracterización de los conocimientos profesionales. Su valor se hace patente cuando nos damos cuenta de los supuestos sobre conocimientos que contradice.

Así, se ha pensado durante mucho tiempo en las investigaciones sobre el pensamiento de los profesores que éstos clasificaban a sus alumnos en una cantidad fija y limitada de tipos (el trabajador pero incapaz, el payaso del curso, etc.). Esta suposición se apoyaba en viejos estudios sobre la imagen que los profesores tienen de sus alumnos; pero nuevos trabajos apuntan más bien a una dependencia del número de categorias respecto at momento y finalidad de la clasificación del alumno por el profesor. Parece prometedor imaginarse como esquema, por ejemplo, los conocimientos profesionales usados en el comienzo de cada clase. El profesor experimentado dispone de más «esquemas de inicion -de modo similar al jugador de ajedrez experimentado- y estos esquemas son más ricos en cuanto a posibilidades de desarrollo en caso de dificultades inesperadas. Semejante imagen parece más adecuada que considerar los conocimientos profesionales como un sistema de reglas o un edificio teórico. Bajo las nociones de los profesionales con experiencia, como por ejemplo cuando hablan de un «comienzo motivador", se esconde presumiblemente un abanico de posibles disefios del inicio de una clase. Aquí falta todavía investigación empírica que debería también analizar las fronteras de la flexibilidad de estos esquemas, es decir cuestionar si la experiencia profesional sólo enriquece o también inflexibiiiza estos esquemas.

\section{NOTA FINAL}

La presentación de los contenidos de los conocimientos profesionales dio como resultado que incluso el aparentemente tan homogéneo «contenido de la asignaturas implica diversos tipos de conocimientos profesio- nales. Naturalmente son admisibles otras programaciones, pero cada programación individual surge mediante la transformación constructiva y sopesada de los conocimientos del profesor sobre la asignatura. No son simplemente versiones diluidas y en sucio de los conocimientos de la especialidad, este trabajo de transformación forma parte de las tareas profesionales de! profesor. Las dificultades de esta tarea son evidentes, dadas las características psicológicas de los conocimientos profesionales. La reflexión sobre las diferencias entre conocimientos teóricos y profesionales puede contrubuir a desmostar ciertas expectativas poco realistas acerca de la inmediata aplicabilidad de la «teoria» y a considerar e investigar el trabajo de los profesores como un oficio.

\section{Agradecimientos:}

Por sus observaciones, doy las gracias a Bernhard Andelfinger, Gudrun Dobslaw und Jörg Voigt.

\section{Notas:}

1. Las expectativas de los profesionales respecto a las teorias y las concepciones sobre aplicabilidad de las teorias científicas todavía no han sido estudiadas. Es éste un campo que necesita nuevos estudios empiricos. Entonces se podría comprobar si la especialidad estudiada y las concepciones respecto a la aplicabilidad de las teorías están interrelacionadas. Así como se ha estudiado con éxito la filosofía cotidiana, sería también posible estudiar las concepciones científicas.

2. La consideración científíco-lógica de la relación entre teorias y las llamadas reglas tecnológicas, tal como se llevó a cabo en el mencionado debate sobre tecnología de la psicología y ciencias de la educación, no abarca todos los aspectos del problema teoria práctica. Asi, existen concepciones cientificas que quieren reducir la diferencia entre conocimientos teóricos y prácticos mediante cambios del nivel de comprensión y de la metodologia que fundamenta la validez de las afirmaciones científicas. Un ejemplo de ello es la «investigación-acción?. De cualquier modo, también en estas formas de cultivar la ciencia, permanece la diferencia de principio lógicamente fundamentada, entre conocimientos cientificos y prácticos.

3. La cuestión de qué conceptos psicológico-cognitivos (esquemas, prototipos, modelos mentales, etc.) son realmente válidos para describir la singularidad de las representaciones mentales en los conocimientos profesionales no puede discutirse aqui y constituye un problema poco trabajado hasta ahora. En la investigación cognitiva sobre profesores se encuentran principalmente elecciones a priori más o menos infundadas sobre determinados conceptos. El concepto de esquema sólo es usado aquí para caracterizar las cualidades del conocimiento humano por él resaltadas.

\section{REFERENCIAS BIBLIOGRAFICAS}

BOMME, R., 1981, Das Denken von Lehrern bei der Unterrichts-vorbereitung. Eine empirische Untersuchung zu kognitiven Prozessen von Mathematiklehrern. (Beltz: Weinheim).

BEN-PERETZ, M., BROMME, R. \& HALKES, R. (Eds.) 1986, Advances of research on teacher thinking. (Swets \& Zeitlinger. Lisse, NL). 
BERLINER, D., 1986, In pursuit of the expert pedagogue. Paper presented at the annual meeting of AERA.

BROMME, R. 1987a, Der Lehrer als Experte - Skizze eines Forschungsansatzes. In: Neber H. (Hrsg.): Angewandte Problemlösepsychologie. (Aschendorff: Münster), 127-151

BROMME, R., 1987b, Teachers' recall on students' difficulties and progress in understanding in the classroom. In Calderhead, J. (Ed.): Exploring Teachers' Thinking. (Holt-Rinehart \& Winston: Eastbourne) (en imprenta).

BROMME, R. \& BROPHY, J. 1986, Teachers' cognitive activities In: Christiansen, B., Howson, G. \& Otte, M. (Eds.): Perspectives on Mathematics Education. (Reidel: Dordrecht, NL). 99-139.

BROMME, R. \& HÖMBERG, E., 1976, Einführende Bemerkungen zum Problem der Anwendung psychologischen Wissens (Technologieproblem). Materialien und Studien Vol. 4 (Institut für Didaktik der Mathematik der Univer sität Bielefeld: Bielefeld).

BROMME, R. \& JUHL, K., 1987, How teachers construe pupil understanding of tasks in mathematics: Relating the content to cognitive processes of the learner. Journal of Curriculum Studies (in press).

BROMME, R. \& SEEGER, F., 1979, Unterrichtsplanung als Handlungsplanung - Eine psychologische Einführung in die Unterrichtsvorbereitung. (Sc1iptor: Königstein).

BUNGE, M., 1967, Scientific research. Vol II: The search for truth. (Springer: Berlin, New York).

BROUSSEAU, G., DAVIS, R.B. \& WERNER, T., 1986, Observing students at work. In: Christiansen, B., Howson, G. \& Otte, M. (Eds.): Perspectives on Mathematics Education. (Reidel: Dordrecht), 205-241.

CALDERHEAD, J., 1984, Teachers' Classroom Decision Making. (Holt, Rinehart \& Winston: London).

CHEVALLARD, Y., 1985, La transposition didactique. (La Pensée Sauvage: Grenoble).

CHI, M. \& GLASER, R., 1986, Problem-solving ability. In: Sternberg, R.J. (Ed.): Human abilities. An information processing approach. (Freeman: New York), 227-250.

CLARK, C.M. \& PETERSON, P.L., 1986, Teachers' Thought Processes. In: Wittrock, M.C. (Ed.): Handbook of research on teaching, thirdedition (Macmillan: New York), 255-296.

DRERUP, M. \& TERHART, E., 1979. Wissensproduktion und Wissensanwendung im Bereich der Erziehungswissenschaft. Zeitschrift fïr Pädagogik, 25, 377-394.

GAGE, N.L., 19794. The scientific basis of the art of teaching. (Teachers College Press: New York).

GIL, D. \& CARRASCOSA, J., 1985, Science learning as a conceptual and methodological change. Eur. Journal of Science Education, 7, 231-236.

HALKES, R. \& OLSON, J.K., (Eds.), 1984, Teacher Thinking: A new perspective on persisting problems in education. (Swets \& Zeitlinger: Lisse, NL).

HERRMANN, T., 1979. Pädagogische Psychologie als psychologische Technologie. In: Brandstätter, J. Reinert,
G. \& Schneewind, K.A. (Hrseg.): Probleme und Perspektiven der pädagogischen Psychologie. (Klett: Stuttgart).

HOFER, M., 1986. Sozialpsychologie erziehersischen Handelns. (Hogrefe: Göttingen).

JECKER, J.D., MANOBY, N. \& BREITROSE, U.S., 1965, Improving accuracy in interpreting non-verbal uses of comprehension. Psychology In the Schools, 2, 239-244.

KOUNIN, J.S., 1970, Discipline and group management in classrooums. (Holt, Rinehart \& Winston: New York).

KRAPP, A. \& HEILAND, A., 1986, Wissenschaftstheoretische Grundfragen der Pädagogischen Psychologie. In: Weidenmann, B. \& Krapp, A., (Hrsg.) Pädagogische Psychologie - Ein Lehrbuch. (Psychologie Verlags Union: München), 41-72.

MARCELO, C., 1986, Teacher planning and interactive toughts of preservice and inservice teachers. In: Lowyk, J. (ed.): Teacher thinking and profesional action. Proceedings of the third ISATT Conference. (University of Leuven: Leuven), 159-173.

MEYER, H.L., 1980, Leitfaden zur Unterrichtsvorbereitung. (Scriptor: Königstein).

POLANYI, M., 1986, Implizites Wissen. (Suhrkamp: Frankfurt).

PRIM, R. \& TILMANN, H., 1973, Grundlagen einer kritisch, rationalen Sozialwissenschaft. (Quelle und Meyer: Heidelberg).

SCHÖN, D., 1983, The reflective practitioner, (Basic Books: New York).

SCHÖNWÄLDER, M.G., 1983, Lehrerarbeit - Arbeit ohne Theorie. Forschungsberichte der Max-Traeger-Stiftung Nr. 14. (Carl-Winter: Heidelberg).

SCHWAB, J.J., 1973, The practical: Arts of eclectic. School Review, 1971, 79, 493-542.

SHULMAN, L., 1986, Those who understand: Knowledge growth in teaching. Educational Researcher, 15, 4-21.

SHROYER, J.C., 1981, Critical moments in the teaching of mathematics: What makes teaching difficult? Dissertation, Michigan State University (Mimeo) East Lansing, Michigan, USA.

THOMPSON, A.G., 1984, The relationship of teachers' conceptions of mathematics and mathematics teaching to instructional practice. Educational Studies in Mathematics, $15,105-178$.

VEENMAN, S., 1984, Perceived problems of beginning teachers. Review of Educational Research, 54, 143-178.

VILLAR ANGULO, L.M., 1986, Pensamientos de los profesores y toma de decisiones. (Servicio de publicaciones de la Universidad de Sevilla: Sevilla).

WAHL, D., SCHLEE, J., KRAUTH, J. \& MARECK, J. 1983, Naive Verhaltenstheorie von Lehrern. (Zentrum für pädagogische Berufspraxis Universität Oldenburg: Oldenburg).

YINGER, R.J., 1980, A study of teacher planning. The Elementary School Journal, 80, 107-127. 